\title{
UN ACTE DE VENTE ARABE PORTANT SUR LA RÉGION D'AHLAT AU VIIe/XIIIe SIÈCLE
}

\author{
par D. SOURDEL et J. SOURDEL-THOMINE
}

(Paris)

Grâce au hasard qui avait jadis permis aux parchemins et papiers anciens de la grande mosquée de Damas, échappés à l'incendie de 1893, d'être transportés à Istanbul où ils sont aujourd'hui déposés au musée des Arts turc et islamiques ${ }^{1}$, divers documents arabes touchant l'histoire religieuse, économique et sociale au moyen âge ont été signalés, ou même déjâ publiés et traduits ${ }^{2}$, dont il n'est point nécessaire de souligner davantage l'intérêt historique, à certains égards exceptionnel. Rares sont en effet, aux époques qu'ils concernent, les pièces d'archives authentiques sur lesquelles on puisse se fonder pour compléter les données des sources littéraires et y découvrir notamment quelques-uns de ces renseignements touchant la vie pratique ou quotidienne qui sont restés le plus souvent étrangers aux curiosités des chroniqueurs médiévaux. La petite série de textes de ce genre conservés, pendant des siècles, dans la grande mosquée de Damas, avec les débris de manuscrits sur parchemin ou papier au milieu desquels ils se trouvent encore, constituent à cet égard une heureuse exception.

Ces quelques pièces d'archives - pour autant que l'on puisse se fier aux premiers résultats des enquêtes auquel nous nous sommes livrés depuis que

1 Sur l'importance de cette collection, voir J. SOURDEL-THOMINE et D. SOURDEL, Nouveaux documents sur l'histoire religieuse et sociale de Damas au moyen-âge, dans Revue des Etudes isl., XXXII, 1964, p. 1-25, et A propos des documents de la grande mosquée de Damas conservés à Istanbul. Résultats de la seconde enquête, dans REI, XXXIII, 1965, p. 73-85. Les conditions dans lesquelles ces parchemins et papiers avaient été sauvés de l'incendie, gardés d'abord à Damas et transportés ensuite à Istanbul, sont signalés notamment dans REI, XXXII, 1964, p. 1-2, et XXIII, 1965, p. 84-85.

2 Voir outre les inventaires partiels et les remarques d'ensemble contenus dans les deux articles cités supra, n. 1, J. SOURDEL-THOMINE et D. SOURDEL, Trois actes de vente damascains du début du IVe/Xe siècle, dans Journal of the Econ. and Soc. and Soc. Hist. of the Orient, VIII, 1965 p. 164-185, ainsi que D. SOURDEL, Un pamphlet musulman anonyme d'époque 'abbāside contre les chrétiens, dans REI, XXXIV, 1966, p. 1-34. 
nous a été ouvert l'accès à cette intéressante collection ${ }^{3}$ nous renseignent essentiellement sur la société damascaine ancienne ou tout au moins syrienne, dans la majorité des cas sur Damas même et ses alentours. Le fait est normal puisque le dépôt d'où elles proviennent avait été situé à l'origine à Damas. Mais il nous a paru d'autant plus digne de remarque de découvrir dans cet ensemble un acte de vente portant sur des terres non pas syriennes mais anatoliennes, dans cette région du lac de Van et de la haute vallée de l'Euphrate où s'était précisément déroulée la bataille de Manzikert dont on célèbre en ce moment l'anniversaire. L'acte lui-même est postérieur à l'occupation turque et mérite par sa date l'appellation de post-seldjoukide. Il se signale à la fois par ses grandes dimensions (rouleau de papier bruni de $102 \times 17,5 \mathrm{~cm}$ ), par l'élégance de son écriture pourtant cursive et rapidement exécutée (absence de points diacritiques et nombreuses ligatures par exemple) ainsi que par son fort bon état de conservation en dépit de quelques taches et de quelques parties effacées $^{4}$ (V. Pl. O)

Surprenante au premier abord, l'existence d'un tel acte de vente parmi des documents typiquement damascains s'explique néanmoins fort bien. A la date précise où il fut rédigé, en $628 / 1231$, le territoire d'Ahlat, qui était resté sous la domination d'une dynastie turque vassale des Seldjoukides de Rum, les Sāh-Arman, entre $493 / 1100$ et $604 / 1207$, se trouvait depuis quelques années aux mains de divers princes ayyoubides. Plus précisément la ville, qui avait été, l'année précédente, envahie par le Hुwārizmšāh Ğalāl aldīn Manguberti, l'occupant et la ravageant à la suite d'un siège de six mois, venait d'être reprise par l'Ayyoubide al-Malik al-Ašraf et reconnaissait donc l'autorité d'une dynastie syrienne ${ }^{5}$. Cette situation qui n'allait pas durer-puisque le Seldjoukide de Rum 'Alä’ al-dīn Kayqubād Ier, allié d'abord aux Ayyoubides pour triompher du Hुwārizmšāh, s'emparait à son tour d'Ahlat et de la région du lac de Van en 630/1233- permet à la fois de comprendre qu'un personnage de nom arabe se soit porté en $628 / 1231$ acquéreur d'une exploitation agricole

3 Nos remerciements les plus vifs s'adressent ici, une fois encore, à M. Can KERAMETLI, directeur du Musée des Arts turcs et islamiques à Istanbul, et à tout le personnel de son musée dont l'obligeance à notre égard ne s'est jamais démentie au cours de ces derniéres années. Qu'ils veuillent bien croire, les uns et les autres, à l'expression de notre gratitude.

4 Ce document avait été déjà signalé, avec une simple allusion à son contenu, par J. SOUR DEL-THOMINE et D. SOURDEL, A propos des documents de la grande mosquée de Damas, dans REI, XXXIII, 1965, p. 80 (document $n^{\circ}$ 5).

5 Sur ces faits bien connus, voir le résumé et les indications bibliographiques contenues dans Enc. Islam, 2 e éd., s. Akhläț ((art. de V. MINORSKY). 
dans les environs d'Ahlat et qu'il ait ensuite regagné Damas en emportant vraisemblablement avec lui le texte de l'acte portant la preuve de son acquisition.

L'acte en question, rédigé en milieu ayyoubide mais concernant une terre anatolienne, unique donc à notre connaissance pour l'époque et le lieu, fournit des informations d'autant plus présieuses -en dépit de leur portée limitéeque la région à laquelle il s'applique a toujours fait preuve d'une réelle originalité due aux conditions géographiques la distinguant des steppes mésopotamiennes et syriennes voisines comme aux effets de conditions historiques également particulières. Très tôt tout d'abord, après le premier essor de l'islam, le haut pays arménien avait été soumis à des princes arabes y régnant en alternance avec des princes locaux, selon les vicissitudes du combat latent poursuivi, sur leurs confins, par les deux empires rivaux de Byzance et de Bagdad. Puis les problèmes qu'y avait dû poser la coexistence entre populations conquises et occupants arabo-islamiques avaient été encore aggravés par les. modalités de la conquête seldjouqide, lorsque la bataille de Manzikert eut ouvert l'Anatolie Orientale aux bandes turcomanes en même temps qu'elle intégrait ce territoire au nouvel empire d-Alp Arslan. Sans doute cette conquête avait-elle eu des répercussions profondes, faisant du pays, à la veille de l'occupation mongole et avant même qu'il entrât officiellement dans le domaine des Seldjoukides de Rum, une province dont l'état économico-social correspondait dans ses grandes lignes à celui qui existait dans les régions occidentales de l'Anatolie. Elle n'avait pu toutefois empêcher le maintien de particularismes locaux dont il est permis de chercher témoignage dans un acte de vente privé, passé entre des personnages qu'aucun détail historique ne signale à notre attention mais qui étaient vraisemblablement, d'après la teneur même de l'acte, arabisés et islamisés de longue date.

Nous nous trouvons de fait en présence d'un achat fait par un notable du nom de Muhammad b. al-Ḥasan b. Muhammad al-Salmānī où l'on peut voir un marchand d'après son titre de hawāğa. Il achète pour son épouse du nom de Humayyita ou Hamatiyya ${ }^{6}$ avec son argent à elle, la propriété d'une autre femme de condition libre. Cette dernière, Tāğ Mulk hātun, ${ }^{7}$ est présentée comme une petite-fille et arrière-petite-fille de șāhib, aux noms arabes et

6 Le nom reste donteux, mais il n'existe actuellement à notre connaissance aucun nom féminin arabe nous paraissant correspondre au ductus lisible sur le document.

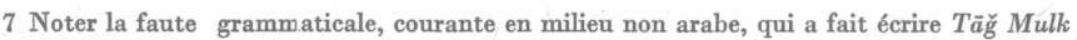
pour Tāğ al-Mulk. 
surnoms musulmans en din, personnages qui ne nous sont pas connus par ailleurs mais dont on sait que le titre, très répandu à cette époque et attesté pour de hauts fonctionnaires comme les vizirs, $n$ ' était cependant pas réservé à ces derniers. ${ }^{8}$ Sans doute s'agissait-il ici d'une dynastie locale de petits seigneurs.

L'objet de la transaction est constitué par une part de domaine agricole, le trentième exactement du “demi-village, d'Arnīs "dans le district, de Hilāṭ", localité d'Arnīs aujourd'hui peu importante mais dont le nom s'est conservé sous la forme Ernis portée sur les cartes et dont l'identification est assurée par le voisinage du village de Suğurt, correspondant au Suburt de notre document. ${ }^{9}$ La permanence de ces deux toponymes est intéressante puisqu'elle situe avec précision le domaine objet de la transaction et qu'elle permet d'attribuer, aux terres du village d'Ernis telles qu'elles apparaissaient au VIIe/XIIIe siècle, une valeur globale approximative de 120.000 dirhams rümĩ, obtenue en multipliant par rente, puis par deux, la somme payée pour la part ici concernée. Il nous faut aussi noter au passage le mode de division du territoire du "demivillage" en trente parts, selon un systéme qui n'est pas usuel dans les pays arabes et qui correspond sans doute à une coutume locale.

La part en question était essentiellement représentée par une "maison d'exploitation agricole" -s'il faut bien interpréter ainsi l'expression arabe bayt filāha utilisée dans le document:, désignée par le nom d'un certain Babtiyār ibn Sīmāfūn (?), nom pour lequel il est impossible de proposer une lecture définitive mais qui semble en tout cas de caractère ni arabe ni turc. ${ }^{10} \mathrm{On}$ pourrait donc penser à un domaine jadis possédé par un autochtone et dont la nouvelle aristocratie locale s'était emparé, peut-être d'ailleurs depuis fort longtemps. Le dit domaine comprenait des terres diverses, irriguées ou non, cultivées ou non, situées en terrain plat ou montagneux - tout ceci d'après les termes même du document -, ainsi que des pâturages (murüğ), qui devaient constituer sa principale richesse dans une région élévée et de climat rude où les meilleures ressources sont encore aujourd'hui fournies par l'élevage. Le

8 C'est ce qui semble ressortir des remarques de Cl. CAHEN, pre-Ottoman Turkey, Londres, 1968 , p. 238 et 242

9 Ernis est située entre Tatvan et Ahlat, dans l'intérieur des terres et dépend actuellement du district de Suğurt. Nous devons cette indication à M. et Mme N. Beldiceanu que nous sommes heureux de remercier ici.- Les autres toponymes mentionnés dans l'acte à propos des limites du domaine n'ont pu être identifiés et n'ont été lus-quand une lecture paraissait possibleque de façon hypothétique.

10 Le premier terme serait plutôt iranien de consomance et le patronyme Sĩmäfūn évoquerait plutôt un nom grec, non identifié exactement. 
fait important à noter est que cet élevage était pratiqué en disposant d'installations fixes même si celles-ci étaient sans doute rudimentaires. Le document spécifie en effet que l'exploitation agricole englobait, outre ses terres et ses pâturages, des éléments utilitaires variés tels ces maqãt et mahṣad al-hašiš qu'il nous a semblé permis d'interpréter comme des étables dans un cas et des granges à fourrage de l'autre. ${ }^{11}$

Mais pour cette exploitation agricole, nous ne disposons que d'une estimation toute relative puisque le dirham rümī, monnaie utilisée dans le texte, ne nous est point connue par ailleurs et nous reste donc de valeur non identifiable. Tout au plus, son nom même prouverait-il que la région d'Ahlat se trouvait, à cette époque, dans l'orbite économique de l'empire saldjoukide de Roum même si un ayyoubide y régnait.

Ajoutons encore que le personnage chargé de faire comparaître les témoins de l'acte et mentionné dans les diverses signatures apposées à la fin du rouleau mérite de retenir l'attention par son abondante titulature le présentant comme le grand cadi ( $q a \bar{a} d \tilde{i} l$ l'quḍât) Tāğ al-milla wa-l-dīn ḥuğğat al-islām wa-l-muslimīn Abū l'Fatḥ Muḥammad b. Saẽid b. Muuhammad al-Hāšimī. De telles épithètes, fréquemment utilisées à l'époque (12), soulignent la relative importance du personnage mais nous ne savons de quel cadi exactement il s'agissait, sans doute le cadi suprême de la principauté ayyoubide qui dominait alors la région d'Ahlat. Il est assez remarquable qu'un hasimite non, connu malheureusement par ailleurs ait occupé pareil poste dans cette région lointaine et à un moment où les hommes de religion d'origine "orientale" étaient de plus en plus nombreux.

Tel paraît donc être le bilan des quelques informations originales contenues dans un texte dont on ne manquera pas de signaler d'autre part la forme longue et le style juridique particulièrement soucieux du moindre détail. Il ne saurait être question de chercher, dans ce document unique, la solution des délicats problèmes que pose le statut des terres dans les pays soumis au régime seldjouqide. On remarquera toutefois que nous ne sommes pas ici en face d'une

11 La traduction "étables" pour le terme maqãt reste hypothétique dans la mesure ou elle n'est pas attestée par les dictionnaires. Le sens tiré de la racine $Q W T$ nous semble désigner les petits abris grossièrement construits, dont il reste des vestiges anciens dans des régions steppiques de climat rude et qui permettaient aux bêtes du même aux gens y trouvant refuge de s'assurer le moyen de subsister.

12 Un grand cadi enterré à Ählat en $667 / 1259$ portait une titulature analogue avec Zayn al-milla wa-1-din; Cf. Répertoire chronologique d'êpigraphie arabe, $\mathrm{n}^{\circ} 4440$. 
terre qui ait pu être considérée comme terre d'Etat. Si, comme on l'a dit, certaines terres anatoliennes furent dès cette époque dotées statut qui se répandra ensuite à l'époque ottomane, nous sommes ici bel et bien en face d'une terre privée qui change de propriétaire selon le procédé normal en pays islamique: terre privée qui appartint à d'anciens habitants d'après le nom resté appliqué au domaine et qui passa ensuite entre les mains de l'aristocratie locale pour être acquise, à la veille de l'invasion mongole, par un marchand apparemment arabe. Faut-il voir là l'indice d'un déclin économique de la classe alors dirigeante, l'indice aussi d'une montée, en contrepartie, de la classe marchande, enrichie par le trafic commercial tout au long des règnes des souverains seldjouqides et inévitablement intéressée à résider dans des régions qui se situaient sur un des axes les plus suivis alors par les courants d'échange du grand négoce. On serait tenté de le croire. Il reste à souhaiter que de nouvelles découvertes viennent un jour confirmer ce qui, en l'état actuel de nos connaissances, demeure seulement une séduisante hypothèse.

\section{TRADUCTION}

Ceci est un écrit où se trouve consigné ce que le marchand Muhammad ibn al-Ḥasan ibn Muhammad al-Salmānī a acheté, pour son épouse de condition libre nommée Humayyita (?) fille de Mạ̣mūd ibn Sa ỉd, avec l'argent de cette dernière et son autorisation, à la [dame) respectable et de condition libre, nommée Tāğ Mulk (sic) Hātūn fille du șāḥib 'Izz al-d.n 'Abd al-Wāḥid fils du șāḥib Niẓām al-dīn Abū Bakr fils du ṣaḥib Muwaffaq al-dīn Abū l-Fatḥ fils de 'Abd al-Wāhịid ibn Rašĩ .

Cette dame a vendu, en état de pleine capacité, ce qui était son droit et son bien, ce qui se trouvait légalement entre ses mains et sous sa gestion jusqu'à la conclusion de cette vente, à savoir une part provenant de la base de trente parts correspondant à toute la moitié, définie par partage, du village appelé Arnīs du district de. Hilāt. Et cette part unique est marquée par la maison d'exploitation de Bahtiyār b. Sīmāfūn (?) [qui est elle-même) contigūë à la maison de Tarāsū (?) ibn.... ${ }^{13} \ldots$ et à celle de Baybars ainsi qu'à la route. [Elle comprend aussi] des terres dépendant de la susdite maison d'exploitation de Bahtiyār, des pâturages, des étables et des granges à fourrage appartenant à la susdite maison de Babtiyār parmi tout ce qui s'ajoute et se rapporte

13 Un mot illisible. 
à cette maison, [tout cela ] compris et contenu entre quatre limites dont la première touche à la limite du village de S. t. r. b. d. (?), la seconde touche à la limite du village de Suburt, la troisième touche au village de Fãmūğ (?) et la quatrième touche à la limite d'Armaknīr (?) avec tout ce que la part unique susdite prise sur la miotié de l'ensemble du village, susdit possède en fait de droits, de limites, de terres irriguées et non irriguées, cultivées et incultes, de plaines et de hauteurs, d'annexes et de dépendances de toutes sortes. [Cette dame l'a vendu pour un prix déterminé d'un montant de deux mille dirhams-fulūs rūmī de bon aloi et ayant cours selon la coutume, dont la moitié... ${ }^{14}$... est de mille dirhams-fulüs rümī ,et ce en vertu d'un achat authentique et légal, d'une vente complète, claire, produisant effet et exécution, contraignante, irrévocable, non susceptible d'annulation, ni d'empêchements... ${ }^{15}$..., ni de clauses trompeuses, si bien que [les deux parties) s'engagèrent dans la procédure de l'acceptation et de la réception légales... ${ }^{16} . .$. , [manifestant leur) satisfaction du contenu d'une manière claire correspondant à leur langage habituel.

En conséquence [ont eu lieu) l'entrée en possession et la remise, respectives et simultanées, de la somme payée et de l"objet de la transaction, [puis) la compréhension complète et suffisante [de ce qu'ils faisaient) par l'une et l'autre des deux parties, la prise de connaissance par ces deux parties, la complète exécution du contrat sans difficultés matérielles ${ }^{17}$ ni légales empâchant la validité de l'acte.

L'objet de la transaction, à savoir une part des trente parts correspondant à la moitié, définie par partage, du village susdit nommé Arnīs, part qui comprend la maison d'exploitation de Baḩtiyār ibn Sīmāfūn (?), ce qui s'y rattache en fait de terres, irriguées ou non, de pâturages, d'étables, de terrains cultivés ou incultes, de granges à fourrage et tout ce qui s'ajoute et se rapporte aux droits et aux limites [de la maison susdite], est entré entre les mains de l'acheteur, le susdit hawāğa Muḥammad ibn al-Ḥasan ibn Muhammad en tant que propriété destinée à celle pour qui est effectué l'achat, à savoir la susdite Humayyita fille de Maḥmūd ibn Sa ĩd, propriété en sa pleine disposition légale.

14. Deux mots illisibles

15 Un mot illisible et trois ou quatre mots manquant.

16 Trois mots illisibles.

17 Traduetion approximative justificé par le contexte, le terme arabe correspendant n'ayant pu être déchifré. 
[La venderesse $]^{18}$ a délivré à l'acheteur et à celle pour qui est effectué l'achat, au sujet de la somme indiquée, à savoir deux mille dirhams-fulūs rümĩ de bon aloi dont la moitié est de mille dirhams-fulūs rūmī de bon aloi, et après versement intégral et complet de cette somme prise par l'acheteur sur les biens de celle pour qui est effectué l'achat, à savoir son épouse Humayyita (?) fille du susdit Mạ̣mūd, une quittance attestant l'intégralité du versement.

L'acte possède effet exécutoire pour tout ce qu'implique sa contrainte légale; la garantie contre toute réclamation et le contrat du vendeur reposent sur la Loi et ses exigences.

Témoignage a été rendu à ce sujet le 13 du mois de Dieu Rağab al-așabb ${ }^{19}$ parmi les mois de l'année 628/17 mai 1231 .

Louange à Dieu et Ses bénédictions sur notre seigneur Muḥammad, sa noble Famille et ses Compagnons purs.

(Suit une série de témoignages rendus à la demande du qãọi quạāt Tāğ al-milla wa-l-dīn huğğat al-islām wa-l-muslimīn Abū l-Fatḥ Muḥammad b. Sa īd b. Muḥammad al-Hāšimī et commençant par la formule ašhadanī mawlānā $q \bar{a}$ dị $l$-quḍāt...)

18 Un mot illisible mais dont la restitution est ainsi exigée par le sens.

19 Epithète du mois de rağab qu'il est difficile de traduire et qui était surtout connue jusqu'ici sous cette forme dans les inscriptions du Maghreb; Cf. G. WIET, Matériaux pour un Corpus Inscriptionum arabicerum, Egypte, II, Le Caire, 1930, p. 37. 
هذا كتاب فيه ذكرما اشترى الخواجة محمد بن الحسن بن محمد السلماني

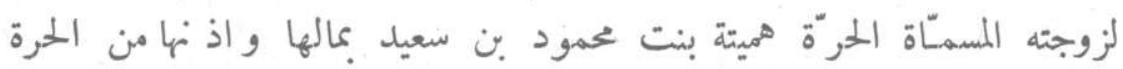

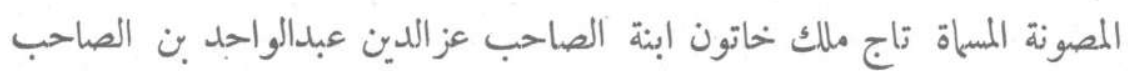

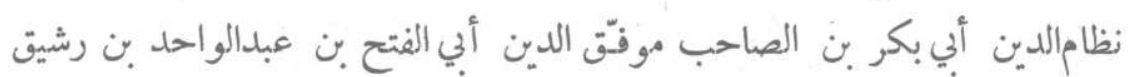

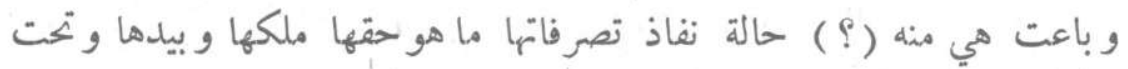

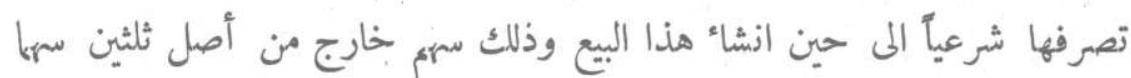

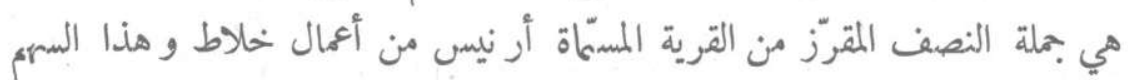

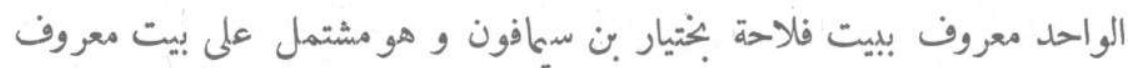

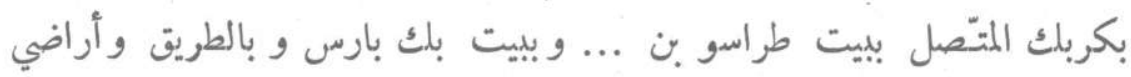

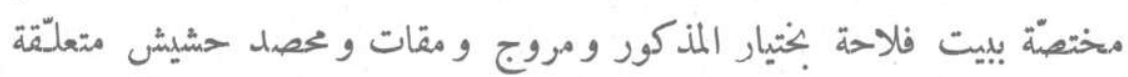

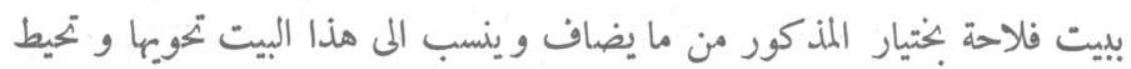

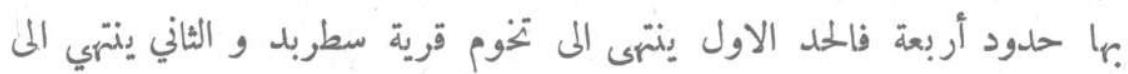

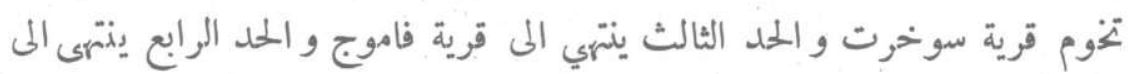

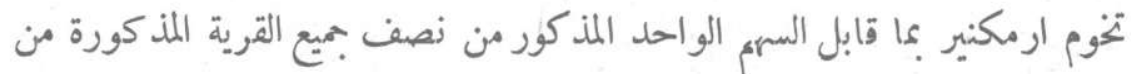

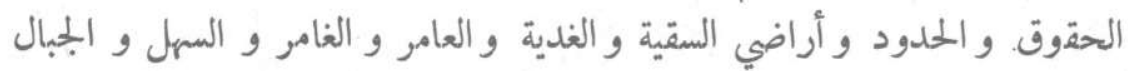

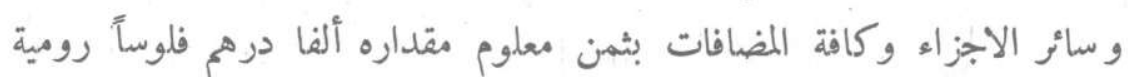

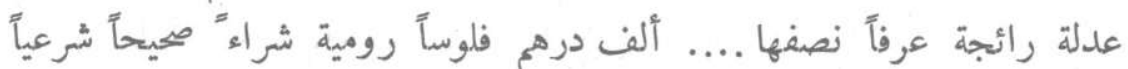

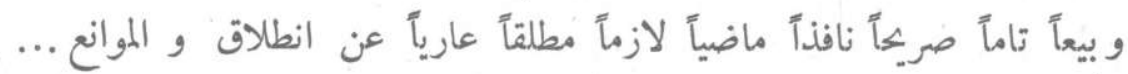

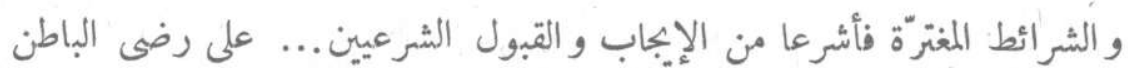

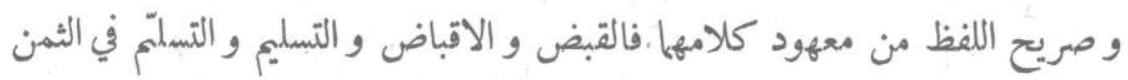


و المثمن جمعيعاً الروبة التامة الكافية من الجانبين و احاطة علمها بذلك و فراغ

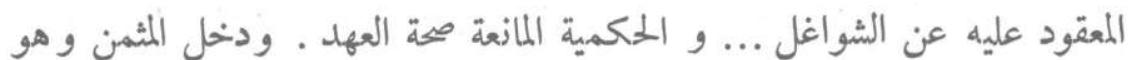
السهم الواحد من أصل ثلثن سهاً "هي جملة النصف المقرّر من جميع القرية المسماة ارنيس المذكور و هذا السهم المذكور هشتمل على بيت فلاحة بختيار بن سيا

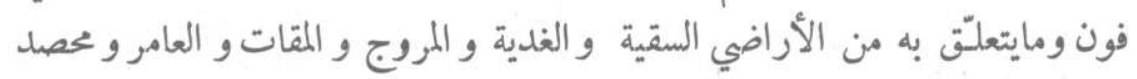

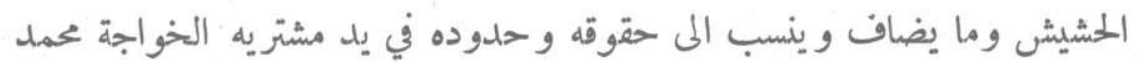

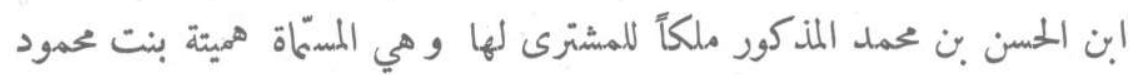

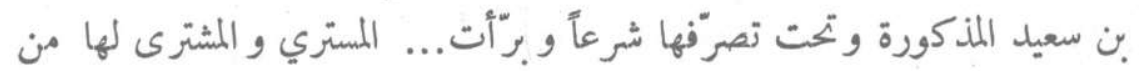

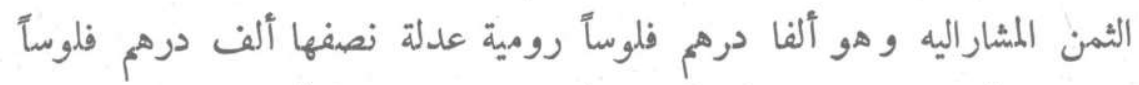

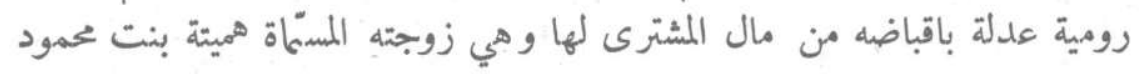

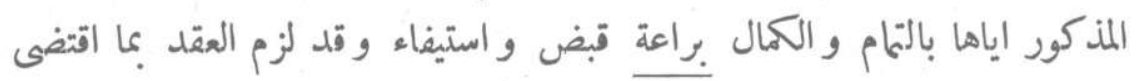

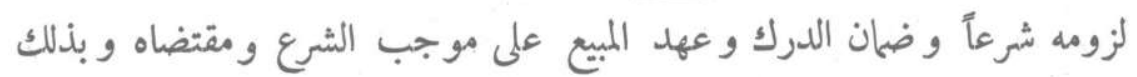

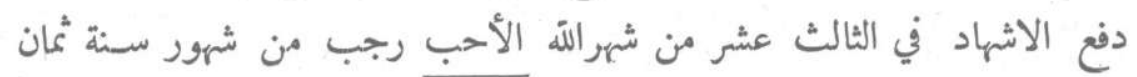
و عشرين و ستمائة و الحمد لله و الصلوة على سيدنا محمد و اله الاكرمين و أصحابه الطاهرين 


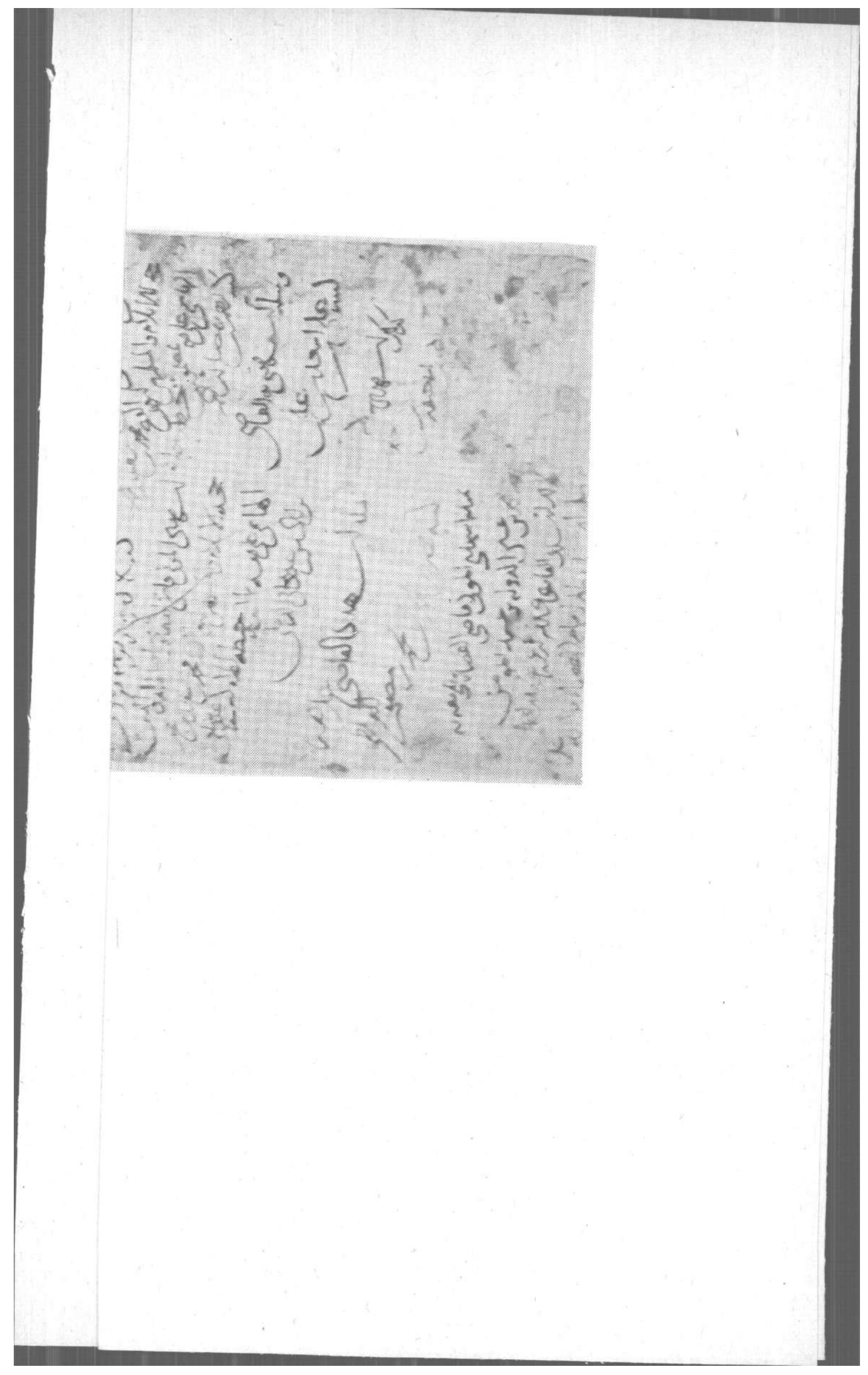

